\title{
Retrospective Evaluation of Instituted Standard Adequacy Criteria for On-Site Adequacy Assessment of Thyroid Fine-Needle Aspiration
}

\author{
Xin Jing, M.D., ${ }^{\star}$ Elizabeth Wey, M.D., and Claire W. Michael, M.D.
}

\begin{abstract}
This retrospective study was conducted to investigate the impact of using instituted standard criteria for on-site assessment of specimen adequacy on fine-needle aspiration (FNA) diagnosis of thyroid nodules. The study included a total of 1,031 thyroid FNAs that were performed and assisted with on-site adequacy assessment using instituted standard criteria from July 2006 to March 2009. Adequate specimens require the presence of at least six groups of follicular cells in total on Diff-Quik stained smears with a minimum of 10 cells in each group. Agreement on specimen adequacy between on-site and final assessment, nondiagnostic rate, distribution of cytologic diagnoses, and cytohistologic concordance for cases with surgical follow-up was evaluated. Implementing the instituted standard criteria resulted in 93\% level of agreement on specimen adequacy between on-site and final assessment. Nondiagnostic rate upon final assessment was $10.7 \%$. Cytohistologic concordant rate reached $93.9 \%$ and $82.3 \%$ for nonneoplastic and neoplastic lesions, respectively. Most importantly, this approach to standardization not only provided diagnostic consistency among cytopathologists, but also minimized confusions and enhanced effective communication. Thus, high satisfactions have been achieved from endocrinologists/radiologists who utilized our on-site assessment service and/or participated in the management of thyroid nodules. Diagn. Cytopathol. 2011;39:391-394. @ 2010 Wiley-Liss, Inc.
\end{abstract}

Key Words: FNA; thyroid nodules; on-site adequacy assessment; instituted standard criteria

Department of Pathology, University of Michigan, Ann Arbor, Michigan

*Correspondence to: Xin Jing, M.D., Department of Pathology, University of Michigan, 1500 E. Medical Center Drive, 2G332 UH, Ann Arbor, MI 48109-0054. E-mail: xinjing@med.umich.edu

Received 1 February 2010; Accepted 5 March 2010

DOI 10.1002/dc.21395

Published online 25 May 2010 in Wiley Online Library (wileyonlinelibrary.com).
It has been well known that various adequacy criteria have been utilized while evaluating permanent smears of fine-needle aspiration (FNA) of thyroid nodules with or without on-site adequacy assessment. ${ }^{1-4}$ Benefits of onsite adequacy assessment for thyroid FNA have been well demonstrated previously. ${ }^{5-7}$ Accordingly, on-site adequacy assessment not only effectively decreased nondiagnostic rate at the time of final cytologic evaluation, but also provided efficient sample triage for ancillary studies. To the best of our knowledge, there are no criteria set forth for on-site adequacy assessment preformed on rapid stained smears. In our institution, the vast majority of thyroid FNAs have been performed with on-site cytopathologist's assistance. The lack of uniform criteria for assessing specimen adequacy resulted in diagnostic inconsistency among the cytopathologists and difficult communications with clinicians. Thus, a standard criteria for on-site adequacy assessment was developed, distributed, and explained to all involved parties including pathologists, endocrinologists, radiologists, and surgeons, and then implemented in our practice in 2005. On-site assessment reports including status of specimen adequacy and provisional diagnosis have been incorporated into our final cytology reports since 2006. The current retrospective study was conducted to assess the impact of using the instituted standard criteria on FNA diagnosis of thyroid nodules.

\section{Materials and Methods}

Through a Computer SNOMED Search from the file at The University of Michigan Hospital between July 2006 and March 2009, we retrieved a total of 1,031 thyroid FNAs with on-site adequacy assessment performed by 
Table I. Agreement on Specimen Adequacy Between On-Site and Final Assessment

\begin{tabular}{lccc}
\hline & \multicolumn{3}{c}{ On-site assessment } \\
\cline { 2 - 4 } Final assessment & $\begin{array}{c}\text { Adequate } \\
n(\%)\end{array}$ & $\begin{array}{c}\text { Inadequate } \\
n(\%)\end{array}$ & $\begin{array}{c}\text { Total } \\
n(\%)\end{array}$ \\
\hline Adequate & $851(99.8)$ & $70(39.3)$ & $921(89.3)$ \\
Inadequate & $2(0.2)$ & $108(60.7)$ & $110(10.7)$ \\
Total & $853(100)$ & $178(100)$ & $1,031(100)$ \\
\hline
\end{tabular}

cytopathologists based on instituted standard criteria. The instituted standard criteria defined specimens as adequate by the presence of at least six groups of follicular cells in total on Diff-Quik (D-Q) stained smears with a minimum of 10 cells in each group. All FNAs were performed under ultrasound guidance by endocrinologists or radiologists. For each pass, a drop was put on a plain slide and smeared by a positively charged slide. One smear was air-dried, stained with D-Q stain and immediately evaluated. The other smear was quickly fixed with Sprayfix ${ }^{\mathrm{TM}}$ and later stained with Papanicolaou stain before final examination. The needle was then rinsed in Cytolyt ${ }^{\circledR}$ solution from which a ThinPrep ${ }^{\mathbb{R}}$ and/or a cell block was prepared as decreed appropriate. At the end of on-site assessment, location of the nodule, estimated size of the nodule, number of passes performed, status of specimen adequacy along with provisional diagnosis (for adequate specimen) was recorded. Both provisional and subsequent final diagnoses were established based on the previously described eight FNA diagnostic categories for thyroid nodules. ${ }^{8}$

Agreement on specimen adequacy between on-site and final assessment, nondiagnostic rate, and distribution of final cytologic diagnoses were evaluated. Further, cytohistologic concordance (neoplasia vs. nonneoplasia) was assessed on 221 cases that were followed up by surgical resection. Cytologic rereview was then performed for cytohistologic discordant cases to investigate causes of the discordance.

\section{Results}

A total of 1,031 aspirates included in the study were prepared from 723 patients (596 female and 127 male). The nodules measured from 0.5 to $10 \mathrm{~cm}$ with median size of $1.9 \mathrm{~cm}$. Number of passes performed ranged from 1 to 12 with a median of six passes.

Table I shows agreement on specimen adequacy between on-site and final assessment. Among 853 specimens that were interpreted as adequate on-site, 851 $(99.8 \%)$ remained concurrent at the time of final assessment while $2(0.2 \%)$ were reversed to nondiagnostic due to on-site misinterpretation of histiocytes as follicular cells. With regard to 178 specimens that were interpreted as inadequate on-site, $70(39.3 \%)$ were converted to adequate specimens upon final assessment because of the presence of additional follicular cells in Papanicolaou stained conventional and/or ThinPrep smears. Final
Table II. Distribution of Cytologic Diagnoses and Cytohistologic Concordance

\begin{tabular}{lccc}
\hline Cytologic diagnosis & $N$ & $\begin{array}{c}\text { Surgical } \\
\text { follow-up N }(\%)\end{array}$ & $\begin{array}{c}\text { Concordant } \\
\text { case } N(\%)\end{array}$ \\
\hline Adequate & 921 & $221(24.0)$ & $139(62.9)$ \\
Non-neoplasia & 722 & $66(9.1)$ & $62(93.9)$ \\
NH & 638 & 62 & $58(93.5)$ \\
LT & 84 & 4 & $4(100)$ \\
FL/HL & 116 & $87(75.0)$ & $21(24.1)^{\mathrm{a}}$ \\
Neoplasia & 83 & $68(81.9)$ & $56(82.3)$ \\
FN/HN & 29 & $19(65.5)$ & $14(73.7)$ \\
PTC & 46 & $41(89.1)$ & $34(82.9)$ \\
Others & 8 & $8(100)$ & $8(100)$ \\
\hline
\end{tabular}

$\mathrm{NH}$, nodular hyperplasia; LT, lymphocytic thyroiditis; FL/HL, follicular/ Hurthle cell lesion of undetermined significance; FN/HN, follicular/

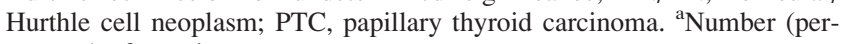
centage) of neoplasm.

assessment identified 921 adequate and 110 inadequate specimens. Consistent results on specimen adequacy (final vs. on-site) were revealed in 851/921 adequate specimens and 108/110 inadequate specimens. Overall, 93.0\% level of agreement (final vs. on-site) on specimen adequacy was achieved. A final nondiagnostic rate was $10.7 \%$.

Distribution of cytologic diagnoses among 921 adequate specimens along with surgical follow-up is presented in Table II. Proportion of nonneoplastic lesions, follicular/ Hurthle cell lesion of undetermined significance (FL/HL), and neoplastic lesions comprising the adequate specimens was: $722(78.4 \%), 116(12.6 \%)$, and $83(9.0 \%)$, respectively. Majority of the nonneoplastic lesions were nodular hyperplasia and less were chronic lymphocytic thyroiditis. Diagnosis of papillary thyroid carcinoma was rendered in more than half $(54.8 \%)$ of neoplastic lesions, followed by follicular/Hurthle cell neoplasm (34.5\%). The remainder $10.7 \%$ was comprised of the less common neoplasms, including medullary thyroid carcinoma, lymphoma, metastatic squamous cell carcinoma, and intrathyroidal parathyroid adenoma. Surgical follow-up was available in $24.0 \%$ of the adequate specimens, including $9.1 \%$ (66/722) of nonneoplastic lesions, 75\% (87/116) of FL/HL, and 81.9\% (68/83) of neoplastic lesions. Rate of cytohistologic concordance (neoplasia vs. nonneoplasia) reached 93.9\% (62/ $66)$ and $82.3 \%(56 / 68)$ for nonneoplastic and neoplastic lesions, respectively. Overall cytohistologic concordance reached $88.1 \%$ (118/134). Among 14 cytohistologic concordant cases that were cytologically diagnosed as follicular/Hurthle cell neoplasm, follow-up histologic examination showed six follicular cell adenomas, two follicular/Hurthle cell carcinomas, and six follicular variant of papillary thyroid carcinomas.

Of 16 cytohistologic discordant cases, slides were available for rereview in 13 cases. Sampling error contributed to four false-negative cases. False-positive results were associated with over-interpretation of nonspecific cytologic findings. For example, cytologic diagnosis of papillary thyroid carcinoma was rendered in four cases 
due to the finding of occasional intra-nuclear grooves, while five cases were misinterpreted as follicular neoplasm due to the presence of a minor population of microfollicles in the setting of predominantly honeycombing tissue fragments, consistent with nodular hyperplasia.

Follow-up histologic examination of $87 \mathrm{FL} / \mathrm{HL}$ revealed $21(24.1 \%)$ neoplasms, including 13 follicular/Hurthle cell adenomas, three follicular/Hurthle cell carcinomas, and five conventional papillary thyroid carcinomas. When the data were reanalyzed including the indeterminate diagnostic category with definitive nonneoplasia and neoplasia categories, and only the 21 histology-confirmed neoplasms were considered cytohistologic concordant, overall cytohistologic concordance dropped to $62.9 \%$ (139/221).

Of the 110 inadequate aspirates, 11 had histology follow-up that revealed eight nodular hyperplasia, one follicular carcinoma, one papillary thyroid carcinoma, and one intrathyroidal parathyroid gland.

\section{Discussion}

To the best of our knowledge, there are no criteria set forth for preliminary on-site assessment of adequacy in FNA cytologic diagnosis of thyroid nodules. Our data demonstrate that implementation of the instituted standard criteria for preliminary on-site adequacy assessment preformed on D-Q stained smears resulted in over 90\% level of agreement on specimen adequacy between on-site and final assessment. Nondiagnostic rate and cytohistologic concordant rate revealed by this study are compatible with that of published literatures. ${ }^{5,9}$ Most importantly, this approach to standardization not only provided diagnostic consistency among cytopathologists, but also minimized confusions and enhanced effective communication. Thus, high satisfactions have been achieved from endocrinologists/ radiologists who utilized our on-site assessment service and/ or participated in the management of thyroid nodules.

With regard to nondiagnostic rate, it has been shown that operator's experience in performing FNA plays an important role. ${ }^{6}$ Since all FNAs included in the current study were performed in a setting of a teaching institution, operator's experience varied, although experienced faculty members routinely performed the first pass and supervised performance of consecutive passes. A few studies have addressed the optimal number of FNA passes for obtaining diagnostic materials with the presence of on-site adequacy assessment. Accordingly, need of four to six passes per thyroid nodules has been suggested in order to establish diagnostic certainty. ${ }^{7,10}$ The vast majority of the FNAs in this study were well-tolerated and the operators routinely performed three or four consecutive passes and then waited for cytopathologist's assessment of adequacy. If needed, additional two to four passes might be performed. Rarely, less than two passes (due to poor tolerance) or more than eight passes were performed. As a result, a median number of six passes per nodule were performed, which is in agreement with the previously reported studies. It is noteworthy to mention that intrinsic nature of thyroid lesions should also be taken into considerations. For example, aspiration of lesions that are mainly composed of colloid or cyst contents may reveal none or less than optimal number of follicular cell. We thus used number of follicular cells as a general but not sole measuring tool while assessing specimen adequacy.

While in the original cytology reports, follicular and Hurthle cell lesion/neoplasm was specified in the diagnosis, Hurthle cell lesion/neoplasm and follicular lesion/neoplasm were grouped into one category in this study as a previous study conducted in our institution has indicated that Hurthle cell lesion/neoplasm did not predict more malignant potential compared to follicular lesion/neoplasm. ${ }^{11}$ Among the cytologically diagnosed follicular/ Hurthle cell neoplasms, follow-up histology revealed follicular variant of papillary thyroid carcinoma in nearly $50 \%$ of the cases. The finding, similarly to previous studies, ${ }^{9,12}$ demonstrate limitations and challenges of utilizing FNA in distinguishing follicular variant of papillary thyroid from follicular neoplasm. Not surprisingly, false-negative and false-positive diagnoses identified in this study were results of sampling error and over interpretation of nonspecific cytologic findings, respectively.

Indeterminate lesion (FL/HL) constituted $11.2 \%$ of all FNAs included in this study. The finding is compatible with the results reported by the other investigators. ${ }^{13,14}$ Histology-confirmed carcinomas were revealed in $9 \%$ cases categorized into the indeterminate group. The data are in agreement with the risk of malignancy $(5-10 \%)$ presented at NCI Thyroid FNA State of the Science Conference. ${ }^{15}$

Carcinomas were identified in 2 of 11 nondiagnostic FNAs that were followed-up by surgical interventions. The result further supports the opinion that nondiagnostic FNAs carry malignant potentials ${ }^{7,16-18}$ and further investigation is needed for these cases.

In summary, this study confirms that specifying six groups of follicular cells as the threshold for adequacy at the time of on-site assessment is appropriate and provides diagnostic accuracy compatible with the literatures. In addition to providing compatible diagnostic accuracy and consistency, our approach to standardization of criteria for preliminary on-site adequacy assessment enhanced effective communication that further resulted in high satisfactions from endocrinologists/radiologists who utilized our on-site assessment service and/or participated in the management of thyroid nodules.

\section{References}

1. Baloch ZW, Tam D, Langer J, Mandel S, LiVolsi VA, Gupta PK. Ultrasound-guided fine-needle aspiration biopsy of the thyroid: Role 


\section{JING ET AL.}

of on-site assessment and multiple cytologic preparations. Diagn Cytopathol 2000;23:425-429.

2. Ylagan LR, Farkas T, Dehner LP. Fine needle aspiration of the thyroid: A cytohistologic correlation and study of discrepant cases. Thyroid 2004;14:35-41.

3. Kelly NP, Lim JC, DeJong S, Harmath C, Dudiak C, Wojcik EM. Specimen adequacy and diagnostic specificity of ultrasound-guided fine needle aspirations of nonpalpable thyroid nodules. Diagn Cytopathol 2006;34:188-190.

4. Mehrotra P, Viswanathan H, Johnson SJ, Wadehra V, Richardson DL, Lennard TW. Ultrasound guidance improves the adequacy of our preoperative thyroid cytology but not its accuracy. Cytopathology 2006;17:137-144.

5. Eedes CR, Wang HH. Cost-effectiveness of immediate specimen adequacy assessment of thyroid fine-needle aspirations. Am J Clin Pathol 2004;121:64-69.

6. Ghofrani M, Beckman D, Rimm DL. The value of onsite adequacy assessment of thyroid fine-needle aspirations is a function of operator experience. Cancer 2006;108:110-113.

7. Zhu W, Michael CW. How important is on-site adequacy assessment for thyroid FNA? An evaluation of 883 cases. Diagn Cytopathol 2007;35:183-186.

8. Jing X, Michael CW, Pu RT. The clinical and diagnostic impact of using standard criteria of adequacy assessment and diagnostic terminology on thyroid nodule fine needle aspiration. Diagn Cytopathol 2008;36:161-166.

9. Sidawy MK, Del Vecchio DM, Knoll SM. Fine-needle aspiration of thyroid nodules: Correlation between cytology and histology and evaluation of discrepant cases. Cancer 1997;81:253-259.
10. Gharib H, Goellner JR. Fine-needle aspiration biopsy of the thyroid: An appraisal. Ann Intern Med 1993;118:282-289.

11. Pu RT, Yang J, Wasserman PG, Bhuiya T, Griffith KA, Michael $\mathrm{CW}$. Does Hurthle cell lesion/neoplasm predict malignancy more than follicular lesion/neoplasm on thyroid fine-needle aspiration? Diagn Cytopathol 2006;34:330-334.

12. Kini SR, Miller JM, Hamburger JI, Smith-Purslow MJ. Cytopathology of follicular lesions of the thyroid gland. Diagn Cytopathol 1985;1:123-132.

13. Greaves TS, Olvera M, Florentine BD, et al. Follicular lesions of thyroid: A 5-year fine-needle aspiration experience. Cancer 2000; 90:335-341.

14. Layfield LJ, Morton MJ, Cramer HM, Hirschowitz S. Implications of the proposed thyroid fine-needle aspiration category of "follicular lesion of undetermined significance": A fiveyear multi-institutional analysis. Diagn Cytopathol 2009;37:710714.

15. Baloch ZW, Cibas ES, Clark DP, et al. The National Cancer Institute Thyroid fine needle aspiration state of the science conference: A summation. Cytojournal 2008;5:6.

16. McHenry CR, Walfish PG, Rosen IB. Non-diagnostic fine needle aspiration biopsy: A dilemma in management of nodular thyroid disease. Am Surg 1993;59:415-419.

17. Chow LS, Gharib H, Goellner JR, van Heerden JA. Nondiagnostic thyroid fine-needle aspiration cytology: Management dilemmas. Thyroid 2001;11:1147-1151.

18. Alexander EK, Heering JP, Benson CB, et al. Assessment of nondiagnostic ultrasound-guided fine needle aspirations of thyroid nodules. J Clin Endocrinol Metab 2002;87:4924-4927. 